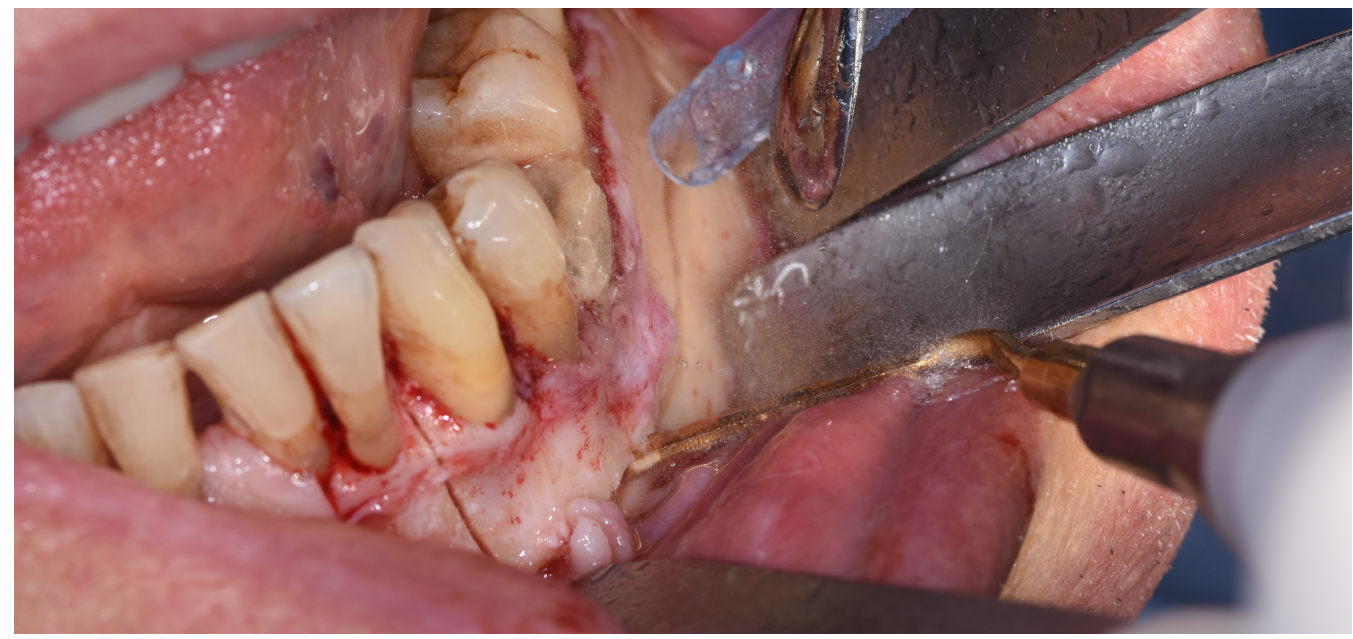

\title{
Safety and efficacy of sectorial resection with piezoelectric device in ONJ
}

Christian Bacci ${ }^{1}$, Mariagrazia Boccuto, Alessia Cerrato, Anna Grigoletto, Gastone Zanette ${ }^{1}$, Annalisa Angelini ${ }^{1}$, Luca Sbricoli

1 University of Padua

Funding: The author(s) received no specific funding for this work.

Potential competing interests: The author(s) declared that no potential competing interests exist.

\section{Abstract}

Aim of the study was to evaluate the efficacy and safety of sectorial bone resection with piezoelectric device in patients with STAGE 1 and 2 (SIPMO/SICMF) ONJ.

Materials and methods: A retrospective study was conducted in cancer and osteometabolic patients with ONJ of Stage 1 or Stage 2 according to SIPMO/SICMF 2.0 who underwent to sectorial bone resection with piezoelectric device and contextual first intention closure.

All procedures were performed in local anesthesia and conscious sedation, in out-patients clinical setting.

Treatment was considered efficient in case of complete epithelization of the site, complete resolution of symptoms and absence of exposure of bone. Piezoelectric device was used without other rotating handpieces.

All patients were followed up at 2 weeks, 8 weeks and 6 months after the surgery.

Results: 10 patients, from 45 to 83 (average 68.4) years old, 7 females and 3 males, were identified.

Bisphosphonates were administrated in 7 cases (zoledronate) for oncological reasons, 2 patients for osteometabolic reasons ( 1 alendronate, 1 hybandronate) while in 1 case for algodystrophy (an off-label use of clodronate).

8 mandibular sectorial osteotomies and 2 maxillary were performed, with a single recurrence. 
3 patients were heavy smokers: this surprising did not compromise the outcome.

Conclusions: According to our case studies the use of piezoelectric device in sectorial bone resection in ONJ seem to be effective and easy to use.

\section{Definitions}

Bisphosphonate related osteonecrosis of the jaw (BRONJ)

Defined by Alberto Bedogni et al.

Medication related osteonecrosis of the jaw (MRONJ)

Defined by Alberto Bedogni et al.

1 Unit of Oral Pathology and Medicine, Section of Clinical Dentistry, Department of Neurosciences, University of Padua 2 Pathology, Department of Cardiac, Thoracic, Vascular Sciences and Public Health, University of Padua

3 Chair of Dental Anesthesia, Section of Clinical Dentistry, Department of Neurosciences, University of Padua

\section{Background:}

Belonging to SIPMO/SICMF 2.0, osteonecrosis of maxillary bones (ONJ) associated with bisphosphonates and/or other drugs is defined as a "presence of necrotic bone or intra/extra oral fistula in the maxillofacial district for more than 8 weeks in patients in therapy with anti-resorptive and/or anti-angiogenic drugs, never subjected to head and neck radiotherapy".

Onj's clinical-radiological staging:

- Stage 1, the presence of at least one minor clinical sign with a bone thickening to computer tomography (CT) limited to the dental-alveolar process only, with or without other early radiological signs;

- Stage 2, the presence of at least 1 minor clinical sign with a bone thickening to CT extended also to the basal process of the mandible or jaw, with or without late radiological signs;

- Stage 3, such as stage 2 with the presence of one or more clinical signs such as extra-oral fistula, spillage of fluids from the nose, preternatural mobility of the jaw with or without preserved occlusion.

Where a minor clinical sign means bad breath, odontogenic abscess, mandibular asymmetry, bone exposure, mucous fistula, mucous hyperemia, failure to repair post extractive alveolar mucosa, rapid-onset dental mobility, paresthesia/lip dysesthesia, purulent secretion, spontaneous seizure of bone fragments, trismus and/or soft tissue swelling.

In CT scan it is possible to identify:

- trabecular thickening, focal medullary osteosclerosis, with or without alveolar crest thickening and hard foil, postextractive alveolar persistence, periodontal space release in stage 1;

- diffuse osteosclerosis, with or without oro-antral and oro-nasal fistula, thickening of the alveolar canal, periostal reaction, 
seizure, sinusitis in stage 2 ;

- muco-cutaneous fistula, pathological fracture, osteolysis extended to the jaw sine, osteosclerosis of cheekbone and/or hard palate in stage 3.

Areas of necrotic bone, regardless of staging of ONJ disease, are a constant source of soft tissue irritation and must be removed without exposure of bone not involved. Sequestra surgery always involves in addition an osteoplasty of the adjacent bone surface to eliminate residual roughness, and the mucous closure first intention of the defect, mobilizing a muco-periosteal flap ${ }^{[1]}$.

\section{Aim of the study:}

Evaluate the efficacy and safety of sectorial bone resection with piezoelectric device in patients with STAGE 1 and 2 (SIPMO/SICMF) ONJ.

\section{Materials and methods:}

A retrospective study (from January 2017 to March 2021) was conducted in cancer and osteometabolic patients with ONJ of Stage 1 or Stage 2 according to SIPMO/SICMF 2.0 who underwent to sectorial bone resection with piezoelectric device and contextual first intention closure.

All procedures were performed in local anesthesia and conscious sedation, in out-patients clinical setting [2]. The surgeries were performed by two different operators.

Treatment was considered efficient in case of complete epithelization of the site, complete resolution of symptoms and absence of exposure of bone. Piezoelectric device was used without other rotating handpieces ${ }^{[3]}$.

For staging and planning the surgeries, each patient was evaluated with panoramic x-ray and CT.

In all cases specimen was sent for histological examination.

Paracetamol 1000 mg, lbuprofen 600 mg as rescue drug amoxicillin and clavulanate 500 mg and metronidazole 250 mg were prescribed.

All patients were followed up at 2 weeks, 8 weeks and 6 months after the surgery.

Privacy was always respected.

\section{Results:}

10 patients, from 45 to 83 (average 68.4) years old, 7 females and 3 males, were identified.

Bisphosphonates were administrated in 7 cases (zoledronate) for oncological reasons, 2 patients for osteometabolic reasons (1 alendronate, 1 hybandronate) while in 1 case for algodystrophy (an off-label use of clodronate).

One of the patients previously in zoledronic acid therapy was in treatment with Denosumab at the time of surgery. 8 mandibular sectorial osteotomies and 2 maxillary were performed, with a single recurrence. 3 patients were heavy smokers: this surprising did not compromise the outcome.

A total of 9 out of 10 interventions were performed by the same operator.

The case of recurrence was followed up. 


\section{Conclusions:}

According to our case studies the use of piezoelectric device in sectorial bone resection in ONJ seem to be effective and easy to use ${ }^{[4]}$.

In 9 cases, no recurrence was observed: complete epithelization of the surgical site, absence of pain, absence of dysesthesia and in some cases improvement of perceived anesthesia or hyperesthesia were noticed.

The relapse found had as its only variable a different operator.

The present case sequence, although limited, converges with the Literature: the mandible $(70 \%)$ is affected more than the maxilla (30\%) [5] [6].

A purpose for the future is to perform a case controlled study to compare different devices in bone segmental osteotomies.

Bisphosphonate related osteonecrosis of the jaw (BRONJ) Medication related osteonecrosis of the jaw (MRONJ)

\section{References}

1. 'Campisi G, Bedogni A, Fusco V. Evoluzione tassonomica di ONJ farmaco-relata. In: Raccomandazioni clinicoterapeutiche sull'osteonecrosi delle ossa mascellari (ONJ) farmaco-relata e sua prevenzione. SIPMO-SICMF, Versione 2.0. 2020 Palermo;:

2. 'G. Manani, C. Bacci, G. Zanette, E. Facco. (2012). Stato attuale della sedazione cosciente in odontoiatria. Dental Cadmos, vol. 80 (7), 357-369. doi:10.1016/j.cadmos.2012.01.004.

3. 'S. Sivolella, G. Brunello, F. Fistarol, E. Stellini, et al. (2017). The bone lid technique in oral surgery: a case series study. International Journal of Oral and Maxillofacial Surgery, vol. 46 (11), 1490-1496. doi:10.1016/j.ijom.2017.06.027.

4. 'Christian Bacci, Stefano Sivolella, Giulia Brunello, Edoardo Stellini. (2014). Maxillary sinus bone lid with pedicled bone flap for foreign body removal: the piezoelectric device. British Journal of Oral and Maxillofacial Surgery, vol. 52 (10), 987-989. doi:10.1016/j.bjoms.2014.04.019.

5. `Ana O Hoff, Béla B Toth, Kadri Altundag, Marcella M Johnson, et al. (2008). Frequency and Risk Factors Associated With Osteonecrosis of the Jaw in Cancer Patients Treated With Intravenous Bisphosphonates. J Bone Miner Res, vol. 23 (6), 826-836. doi:10.1359/jbmr.080205.

6. ^Robert Coleman, David Cameron, David Dodwell, Richard Bell, et al. (2014). Adjuvant zoledronic acid in patients with early breast cancer: final efficacy analysis of the AZURE (BIG 01/04) randomised open-label phase 3 trial. The Lancet Oncology, vol. 15 (9), 997-1006. doi:10.1016/s1470-2045(14)70302-x. 